\title{
Maternal neural responses to infant cries and faces: relationships with substance use
}

\section{Nicole Landi ${ }^{1,2}{ }^{*}$, Jessica Montoya ${ }^{3}$, Hedy Kober ${ }^{3}$, Helena J. V. Rutherford ${ }^{1}$, W. Einar Mencl ${ }^{2}$, Patrick D. Worhunsky ${ }^{3}$, Marc N. Potenza ${ }^{1,3,4}$ and Linda C. Mayes ${ }^{1}$}

1 Yale Child Study Center, Yale University School of Medicine, New Haven, CT, USA

${ }^{2}$ Haskins Laboratories, New Haven, CT, USA

${ }^{3}$ Department of Psychiatry, Yale University School of Medicine, New Haven, CT, USA

${ }^{4}$ Department of Neurobiology, Yale University School of Medicine, New Haven, CT, USA

\section{Edited by:}

Rina Eiden, University at Buffalo, USA

Reviewed by:

Eamon McCrory, University College London, UK

Andrew James Gerber, Columbia

University, USA

*Correspondence:

Nicole Landi, E 74 Yale Child Study Center, 230 South Frontage Road,

New Haven, CT 06511, USA

e-mail: nicole.landi@yale.edu
Substance abuse in pregnant and recently post-partum women is a major public health concern because of effects on the infant and on the ability of the adult to care for the infant. In addition to the negative health effects of teratogenic substances on fetal development, substance use can contribute to difficulties associated with the social and behavioral aspects of parenting. Neural circuits associated with parenting behavior overlap with circuits involved in addiction (e.g., frontal, striatal, and limbic systems) and thus may be co-opted for the craving/reward cycle associated with substance use and abuse and be less available for parenting. The current study investigates the degree to which neural circuits associated with parenting are disrupted in mothers who are substance-using. Specifically, we used functional magnetic resonance imaging to examine the neural response to emotional infant cues (faces and cries) in substance-using compared to non-using mothers. In response to both faces (of varying emotional valence) and cries (of varying distress levels), substance-using mothers evidenced reduced neural activation in regions that have been previously implicated in reward and motivation as well as regions involved in cognitive control. Specifically, in response to faces, substance users showed reduced activation in prefrontal regions, including the dorsolateral and ventromedial prefrontal cortices, as well as visual processing (occipital lobes) and limbic regions (parahippocampus and amygdala). Similarly, in response to infant cries, substance-using mothers showed reduced activation relative to non-using mothers in prefrontal regions, auditory sensory processing regions, insula and limbic regions (parahippocampus and amygdala). These findings suggest that infant stimuli may be less salient for substance-using mothers, and such reduced saliency may impair developing infant-caregiver attachment and the ability of mothers to respond appropriately to their infants.

Keywords: fMRI, emotion, cry, parenting

\section{INTRODUCTION}

In 2007, The National Survey on Drug Use and Health (NSDUH) found that $5.2 \%$ of pregnant women aged $15-44$ years reported using illicit drugs during pregnancy; in addition, $11.6 \%$ reported alcohol use and $16.4 \%$ reported tobacco use. While drug, alcohol and tobacco abuse are a significant public health concern for all individuals, use of these substances during pregnancy and the post-partum period may have particularly detrimental consequences in mothers. This may be both because of the direct impact of teratogenic substances on infant development, and because the effects of drug abuse on maternal behavior and brain function may negatively impact the post-partum parenting environment.

Data indicate that maternal substance use and abuse are associated with poor parenting measures. Substance-abusing mothers have a two-fold increase in the removal of their children from their care (U.S. Dept. of Health and Human Services, National Center for Health Statistics, and National Health Interview Survey,
1999). Interview and self-report assessments reveal differences in child-related attitudes of substance-using mothers compared to non-substance-using mothers; specifically, substance-using mothers demonstrate less understanding about their child's development and use harsher discipline (Mayes and Sean, 2002). Mothers identified as cocaine users during pregnancy were observed as responding more passively and spending more time disengaged from their newborn compared to drug-free mothers (Gottwald and Thurman, 1994). A similar pattern in maladaptive interactions was also observed in substance-using mothers parenting their child beyond infancy and into toddlerhood (Johnson et al., 2002; Molitor and Mayes, 2010).

At a neurobiological level, alterations in reward and motivational circuitry contribute to substance use, abuse and addiction (Volkow and Li, 2004; Everitt and Robbins, 2005). It has been suggested that as a consequence, decisions are made to use substances at the expense of other behaviors (e.g., relating to parenting; 
Chambers et al., 2007). Specifically, in the addictive cycle, the reward system may be "co-opted" for purposes of maintaining habitual use behavior; in this process, other more adaptive rewards may not hold the same value for users if they are not part of the conditioned reward/motivation link associated with substance use. Importantly, these more adaptive rewards include social affiliation and relationships, and this kind of co-optation may have profound implications for parenting behaviors among addicted adults. Moreover, key neural regions associated with motivation and reward, including the prefrontal cortex and amygdala, are also engaged when parents perceive and/or interact with infant cues (for a review of this literature see Rutherford et al., 2011). Thus, we suggest that in addition to overt negative behavioral patterns associated with addiction that may cause difficulty in parenting (e.g., increased irritability), drug, alcohol and tobacco use may have a direct impact on maternal infant interactions in the postpartum and a direct effect on those neural systems that have been identified as important for maternal behavior.

To address this question directly, our current investigation focuses on identifying the differences between substanceusing and non-using mothers in neural circuitry involved in early maternal infant attachment. Specifically, we investigate the neural response to infant expressions of emotion in recently post-partum substance-using and non-substance-using mothers. Infant-caregiver attachment is forged during early development when cries and facial expressions are the primary means of infant communication with their caregiver. The way in which a caregiver interprets and responds to these cues can directly influence the quality of the attachment between caregiver and infant, as evidenced by the adverse developmental outcomes when infantcaregiver interactions are compromised by depression or substance abuse in caregivers (Murray, 1992; Mayes et al., 1997; Network NICHD Early Child Care Research, 1999). In the current study we seek to further investigate the substance-using maternal brain response using fMRI to these early expressions of infant emotion (specifically, cries and facial expressions) relative to non-using mothers during the first three post-partum months.

\section{PREVIOUS fMRI INVESTIGATIONS OF RESPONSE TO EMOTIONAL STIMULI IN SUBSTANCE USERS}

Although there exists a fairly large literature of adult processing of emotional stimuli in both parents and non-parents (reviewed below), there is relatively little extant investigating of processing of human emotional stimuli in substance-using adults. To date, most fMRI work in this area has focused on presenting participants with craving relevant stimuli such as drug paraphernalia. These studies typically identify in association with drug cues increased activation in regions such as the anterior cingulate cortex (ACC), dorsolateral prefrontal cortex (dlPFC), orbiotofrontal cortex, amygdala and temporal regions (Breiter et al., 1997; Maas et al., 1998; Childress et al., 1999; Wexler et al., 2001). With respect to processing emotionally laden stimuli not directly associated with drug use/misuse, one fMRI study (Asenio et al., 2010) showed groups of cocaine-using and non-using subjects images from the International Affective Picture System (IAPS). These pictures are designed to elicit unpleasant, neutral, or pleasant emotions. The data revealed greater activation for controls in the pleasant condition in the superior and inferior frontal lobules (bilaterally) the anterior nucleus of the thalamus, dorsomedial prefrontal cortex (dmPFC), ACC, and right striatum. There were no group differences for negative or neutral stimuli. However, other studies have found relatively diminished activation of cortical and subcortical regions in cocaine dependent versus control subjects during the processing of simulated interpersonal interactions of sad content, with some of the regions overlapping with those relatively overactivated in cocaine dependent subjects in response to drug cues (Wexler et al., 2001). Taken together, these findings suggest that regions that may respond to rewarding stimuli (such as positive emotion or arousal) or to negative emotions (sad interpersonal interactions) are relatively deactivated in substance users compared to non-users, but that some of these regions can be activated to a greater degree in response to craving inducing stimuli.

\section{PREVIOUS FMRI INVESTIGATIONS OF MATERNAL RESPONSE TO INFANT EMOTION (FACES AND CRIES)}

Prior fMRI studies that have examined the neural response to infant cries in parents and have compared cries to non-cry acoustic stimuli (e.g., white noise), and have found greater recruitment of regions associated with auditory processing and emotional processing/regulation during the perception of cries relative to a control sound (Lorberbaum et al., 2002; Seifritz et al., 2003; Swain et al., 2007; Swain and Lorberbaum, 2008). These regions that include the hypothalamus, midbrain, basal ganglia, ACC, prefrontal cortex, and thalamus are common areas of activation associated with parental responses to infant cries in fMRI paradigms, as well as being commonly associated with motivation and reward-processing. Additionally, observed increases in activation in the insula and prefrontal cortex for cries relative to white noise suggest that circuitry associated with social cognition and empathic processes may also be relevant to parental responsiveness to cries. Notably, neural responses to cries appear to be modulated by time since delivery; mothers showed greater cingulate, amygdala, and insula activation at 2-4 weeks post-partum when listening to their own relative to another baby's cry, whereas at 3-4 months post-partum no greater activity was seen in these regions. Instead, increased activity in the medial prefrontal cortex was observed for own baby cry relative to other baby cry (Swain et al., 2003, 2004a). These findings suggest that experience with an infant over the first several months post-partum may influence the neural response to cries, and that this may reflect the functional re-organization of sensitivity to infant cues in parents.

With respect to processing of facial expression/emotion the mesocorticolimbic circuitry has been implicated in maternal responses to infant faces. For example, studies have found increased activity in the striatum, as well as increased activity in the medial prefrontal cortex, occipital cortex, insula, ACC, and in some studies amygdala and parahippocampus, when mothers view images of infants faces (Bartels and Zeki, 2004; Leibenluft et al., 2004; Nitschke et al., 2004; Strathearn et al., 2008). Furthermore, a recent fMRI study identified maternal brain responses to infant facial affective states (happy, neutral, and sad) in dopamine-associated reward-processing areas when first-time mothers viewed images of their own infants compared to unknown infants expressing comparable affective expressions (Strathearn 
et al., 2008). Specifically, these authors found that happy, but not neutral or sad own-infant faces, significantly activated nigrostriatal brain regions interconnected by dopaminergic neurons, including the substantia nigra and dorsal putamen. In addition, a region-ofinterest (ROI) analysis in this region revealed that activation was related to positive infant affect (happy $>$ neutral $>$ sad) for each own-unknown infant face contrast.

The present study extends this work on maternal perception of infant cries and facial expression by examining how substance use status may relate to maternal neural response to audio and visual cues of different infant emotions. Specifically, we examine neural response to cries of varying distress levels and to faces displaying happy, sad or neutral emotion in mothers using cocaine, marijuana, tobacco, alcohol, amphetamines, heroin, opiates, or a combination of these substances (see substance use status below) relative to a group of age-matched mothers who were not users of any of these substances. We include cries of varying distress levels and faces displaying a range of emotion in order to examine systems responsible for distinguishing between different infant emotional states and needs (Wolff, 1969). Given what we know about disruptions in mother-infant interactions in substanceusing populations, we predicted that substance-using mothers (compared to non-substance-using mothers) will show reduced activation in regions previously identified as being relevant for parenting (active while parents view infant faces and listening to infant cries), including sensory processing regions (visual for faces, auditory for cries), emotional processing regions such as the amygdala, insula and striatum, as well as regions involved in cognitive control such as the prefrontal cortex and cingulate.

\section{MATERIALS AND METHODS PARTICIPANTS}

All participants provided informed consent and data were collected approximately 2 months into the post-partum period (range was 1-3 months). Substance use status was determined by a combination of self-report data and urine toxicology (see below). Sixty-two participants were initially scanned (31 substance-using and 31 non-using participants); 8 subjects were excluded due to excessive motion ( 5 substance-using and 3 non-using), leaving 26 substance-using and 28 non-using mothers in the sample.

\section{Substance-using mothers}

Twenty-six-English-speaking, right-handed recently post-partum mothers who used one or more teratogenic substances (see substance use status below) with normal or corrected-to-normal vision, between the ages of 18 and 42 years inclusive $(M=25.58$ $\mathrm{SD}=5.64)$, participated. Racial/ethnic composition included 5 Caucasian, 16 African American, and 5 Hispanic women.

\section{Non-using mothers}

Twenty-eight-English-speaking, right-handed recently postpartum mothers participated. These individuals were free of tobacco or illicit substance use, had normal or corrected-tonormal vision, and were between the ages of 17 and 42 years inclusive $(M=29, \mathrm{SD}=5.89)$. Racial/ethnic composition included 19 Caucasian, 1 Asian American, 5 African American, 1 woman of mixed race (African American and Caucasian) and 2 Hispanic women.

\section{Additional participant information}

With regard to socioeconomic status, we gathered data on maternal education level. All mothers in the non-using group had completed high school and many had completed four or more years of college (mean number of years of education $=17 ; \mathrm{SD}=3.45$ ). In the substance-using group, 12 of the 26 had completed education through to at least high school, with 3 having gone on to college (mean years of education $=12 ; \mathrm{SD}=1.80$ ). Seventy-eight percentage of the non-using participants were first-time mothers and 30\% of the substance-using participants were first-time mothers. The mean number of children in the home for the substance-using group was $2(\mathrm{SD}=0.97)$, and the mean number of children in the non-using group was $1(\mathrm{SD}=0.89)$.

All participants were paid $\$ 80$ and given a small gift for the baby (e.g., baby blanket, baby supplies, baby toy, or baby chair) for their participation. Participants had no neurological impairment or head injury. Written, informed consent was obtained in accordance with the Yale School of Medicine Institutional Review Board. This study has an associated certificate of confidentiality from NIDA.

\section{Substance use status}

Participants were recruited through rehabilitation facilities, maternity wards, and flyers posted on local busses and gathering spots. Substance use status was determined by a combination of self-report data and urine toxicology. Women were considered substance-using if they used any teratogenic substance (including tobacco, heroin, marijuana, opiates, cocaine, alcohol) during pregnancy and/or into the post-partum period (see Table 1). Because some women were in active drug treatment $(N=2)$, they may not have been using at the time of their MRI; however, if they reported substance use, they were included in the substance-using group. Conversely, if participants did not self-report use but tested positive for any of the above named substances they were included in the substance-using group as well (17 of the 26 substanceusing women tested positive (urine toxicity) for one or more substances on the day of the scan). For alcohol and tobacco use, the Fagerstrom test for nicotine dependence (FTND) and alcohol use disorder identification test (AUDIT) were collected. See Table 1 for a breakdown of particular substance use by participant.

\section{STIMULI}

\section{Auditory stimuli}

Cry stimuli were 2-s segments generated from those used by Green and Gustafson (1983). The cries came from infants who ranged in age from 27 to 32 days. All infants were healthy at birth and healthy at their 1-month checkups. Cries were recorded in the infant's home before the infants were fed. Detailed information about the recording procedure has been reported elsewhere (Green and Gustafson, 1983). For the current experiment, we chose four 2-s segments from two different infants that we determined (by two experimenters) to be either of high- or low-distress, resulting in both a high- and low-distress exemplar from each infant. Distress level was verified by an independent group of 10 female participants (ages 19-24; none of the participants had children) who rated the cries for distress level. Participants rated each cry on a scale of $1-10$, with 1 representing "calm" and 10 representing 


\begin{tabular}{|c|c|c|c|}
\hline Substances used & $\mathbf{N}$ & Self-report (\%) & $\begin{array}{l}\text { Positive } \\
\text { toxicology (\%) }\end{array}$ \\
\hline Tobacco only & 10 & 10 & 90 \\
\hline Marijuana only & 4 & 0 & 100 \\
\hline Alcohol only & 2 & 100 & 0 \\
\hline Non-disclosed drugs & 4 & 100 & 0 \\
\hline Heroin, tobacco, and cocaine & 1 & 100 & 0 \\
\hline Alcohol and tobacco & 2 & 100 & 100 \\
\hline $\begin{array}{l}\text { Alcohol, tobacco, and other } \\
\text { non-disclosed drugs }\end{array}$ & 1 & 100 & 0 \\
\hline Tobacco and heroin & 1 & 0 & 100 \\
\hline Amphetamines and tobacco & 1 & 0 & 100 \\
\hline
\end{tabular}

"distressed". High-distress cries were rated as significantly more distressed $(M=8.06, \mathrm{SD}=1.3)$ than low-distress cries $(M=3.54$, $\mathrm{SD}=0.82 ; t=11.52, p<0.001)$. In addition to cries, participants in the MRI study also heard a "neutral" $220-\mathrm{Hz}$ pure tone. Additional information on the acoustic properties of the cries and neutral stimuli are contained in the Appendix.

\section{Visual stimuli}

Photographs of infant faces between the ages of 5 and 10 months were used; these images, initially used by Strathearn and McClure (2002), were modified to include only the baby's head and not the full body. The stimulus set consisted of 21 images from each of six infants, resulting in a total of 126 images. Infant stimuli were balanced for both gender and race and included Caucasian and African American babies. The infant face images displayed affective states of happy, neutral, and sad. The size, luminance, and contrast were kept constant for all face stimuli, and faces were presented on a gray background. Prior to imaging, face stimuli were rated by an independent group of 11 participants who were not mothers on a scale of 1 (happy) to 10 (distressed). A repeated measures ANOVA of the infant face ratings of subjective responses to the three types of emotional facial cues (happy, neutral, sad) was significant $F(2,20)=146.43, p<0.001$. Pair-wise comparisons showed that happy faces $(M=2.19, \mathrm{SD}=0.75)$ were rated as significantly less distressed (Mean difference $=-1.55, \mathrm{SD}=1.15, p=0.006$ ) than neutral faces $(M=3.74, \mathrm{SD}=143)$. Neutral faces were rated as significantly less distressed (Mean difference $=-4.16, \mathrm{SD}=1.28$, $p<0.001)$ than sad faces $(M=7.90, \mathrm{SD}=0.34)$.

\section{DESIGN}

The stimuli were presented using E-Prime software (Version 1.2; Psychology Software Tools Inc., Pittsburgh, PA, USA). The auditory stimuli were delivered via headphones with no visual display. The visual stimuli were displayed foveally at the fixation point for $1000 \mathrm{~ms}$ and followed by a fixation cross. Subjects received seven functional runs, each consisting of 42 trials (six trials of each condition of interest and six one-back memory trials). The conditions of interest were high-distress cry, low-distress cry, neutral tone, happy face, sad face, and neutral face. Trials were presented in a different randomized order in each functional run, with the constraints that the one-back catch trials could not be presented on the first trial, or immediately repeated. The duration of the inter-trialinterval (ITI) was jittered (4000-14000 ms) to allow event-related analysis and to minimize stimulus expectation.

During each run, subjects were asked to attend to the stimulus sequence of faces and cries. For the one-back memory trials ( $14 \%$ of total trials), subjects were presented with a row of question marks and either a visual stimulus (infant face) was presented above the question marks or an auditory stimulus (cry or tone) was delivered via the headphones. The question marks cued the subject to make a yes/no decision via a stimulus response box as to whether the current stimulus was identical to the stimulus of the preceding trial (i.e., a one-back memory task). No action was required of participants on non-catch trials. Analysis of catch trial data revealed an accuracy rate of $84 \%$ correct for non-using mothers and $70 \%$ for substance-using mothers. The rare catch trials were included to enhance and assess subjects' attention during task performance and were modeled but not included in further analyses.

\section{DATA ACQUISITION}

Data were acquired with a Siemens Trio 3T magnetic resonance imaging system (Siemens AG, Erlangen, Germany) using a standard 12-channel head coil. Localizer images were acquired for prescribing the functional image volumes, aligning the eighth slice parallel to the plane transecting the anterior and posterior commissures. Functional images were collected using a gradient echo, echoplanar sequence [repetition time $(\mathrm{TR})=2000 \mathrm{~ms}$; echo time $(\mathrm{TE})=30 \mathrm{~ms}$; flip angle $=80^{\circ}$, field of view (FOV) $20 \mathrm{~cm} \times 20 \mathrm{~cm}$, $64 \times 64$ matrix, $3.4 \mathrm{~mm} \times 3.4 \mathrm{~mm}$ in-plane resolution, $4 \mathrm{~mm}$ slice thickness, 32 slices]. Each stimulus run consisted of 163 volumes, including an initial rest period of $12 \mathrm{~s}$ (to achieve signal stability) that was removed from analyses.

\section{IMAGE ANALYSIS \\ Preprocessing}

Functional data were preprocessed using SPM5 (Wellcome Functional Imaging Laboratory, London, UK), following our prior published methods (e.g., Kober et al., 2010). This included slicetime correction to the first slice of each volume; SPM's twopass realign-to-mean strategy (which ultimately realigns all functional images to a mean functional image); coregistration of the anatomical image and the average of these realigned functional images; coregistration of all functional images using the parameters obtained from coregistration of the mean image; application of the SPM Unified Segmentation process to the anatomical scan, using prior information from the International Consortium for Brain Mapping (ICBM) Tissue Probabilistic Atlas and estimation of non-linear warping parameters (e.g., Ashburner and Friston, 2005); warping the functional images to the Montreal Neurological Institute (MNI) template space; reslicing into isometric $3 \mathrm{~mm} \times 3 \mathrm{~mm} \times 3 \mathrm{~mm}$ voxels; subsequent smoothing of functional images using a $6 \mathrm{~mm}$ isometric Gaussian kernel. Participants who could not be properly warped using the segmentation routine $(N=6)$ were separately normalized to the MNI structural 
brain without segmentation. All images were inspected for motion in excess of one voxel $(3 \mathrm{~mm} \times 3 \mathrm{~mm} \times 3 \mathrm{~mm})$; eight participants were excluded from the analysis for excessive motion (in excess of one voxel).

\section{GLM data analysis}

Once the functional images were preprocessed, first-level robust regression was performed using the standard general linear model but with iteratively reweighted least squares using the bisquare weighting function for robustness (Wager et al., 2005; Kober et al., 2010), as implemented in MATLAB 7.3 (Mathworks, Natick, MA, USA; robust.m). Motion parameters and high-pass filter parameters were added as additional regressors of no interest. Once conditions were estimated using percent signal change for each participant, a second-level, random effects analysis was performed to estimate group activity and to compare activity between-groups, using NeuroElf (NeuroElf.net) and following our prior methods. We then used Monte-Carlo simulation implemented in Alpha Sim to identify voxels that survived whole-brain correction. Clusters were considered significant at a corrected $p<0.05$ threshold at an uncorrected voxel-level threshold of $p<0.005$ at each tail and a cluster of 25. Anatomical labels of all results were confirmed using the Talairach Daemon toolbox as well as manually, using a human brain atlas (Talairach and Tornoux, 1988).

\section{RESULTS}

Our primary interest was to compare activity elicited in response to infant cries and faces in substance-free mothers and substanceusing mothers. We therefore performed between-group comparisons for activity during each condition. Consistent with our hypotheses, we found relatively increased activity in non-using mothers relative to substance-using mothers for both face and cry stimuli. We now discuss the non-using relative to substance-using findings for each condition (faces, cries) in turn.

\section{FACES}

Figures $1 \mathrm{~A}-\mathrm{C}$ shows the contrast of substance non-using $>$ substance-using mothers in response to happy, sad and neutral faces respectively.

\section{Happy expressions}

In response to happy infant faces we observed greater activation for non-using mothers relative to substance-using mothers in prefrontal regions including ventromedial prefrontal cortex (vmPFC), the right dlPFC/middle frontal gyrus (MFG) and $\mathrm{dmPFC}$ (including medial and superior frontal gyri). We also observed greater activity for non-using relative to substance-using mothers in visual processing regions, such as the middle occipital gyrus, as well as in limbic regions including the right hippocampus/parahippocampus as well as in the cerebellum. We observed only one small region of increased activation for substance-using relative to non-using in the left posterior parahippocampal gyrus (see Table 2).

\section{Sad expressions}

In response to sad infant faces, multiple and extensive regions distinguished non-using from substance-using mothers.

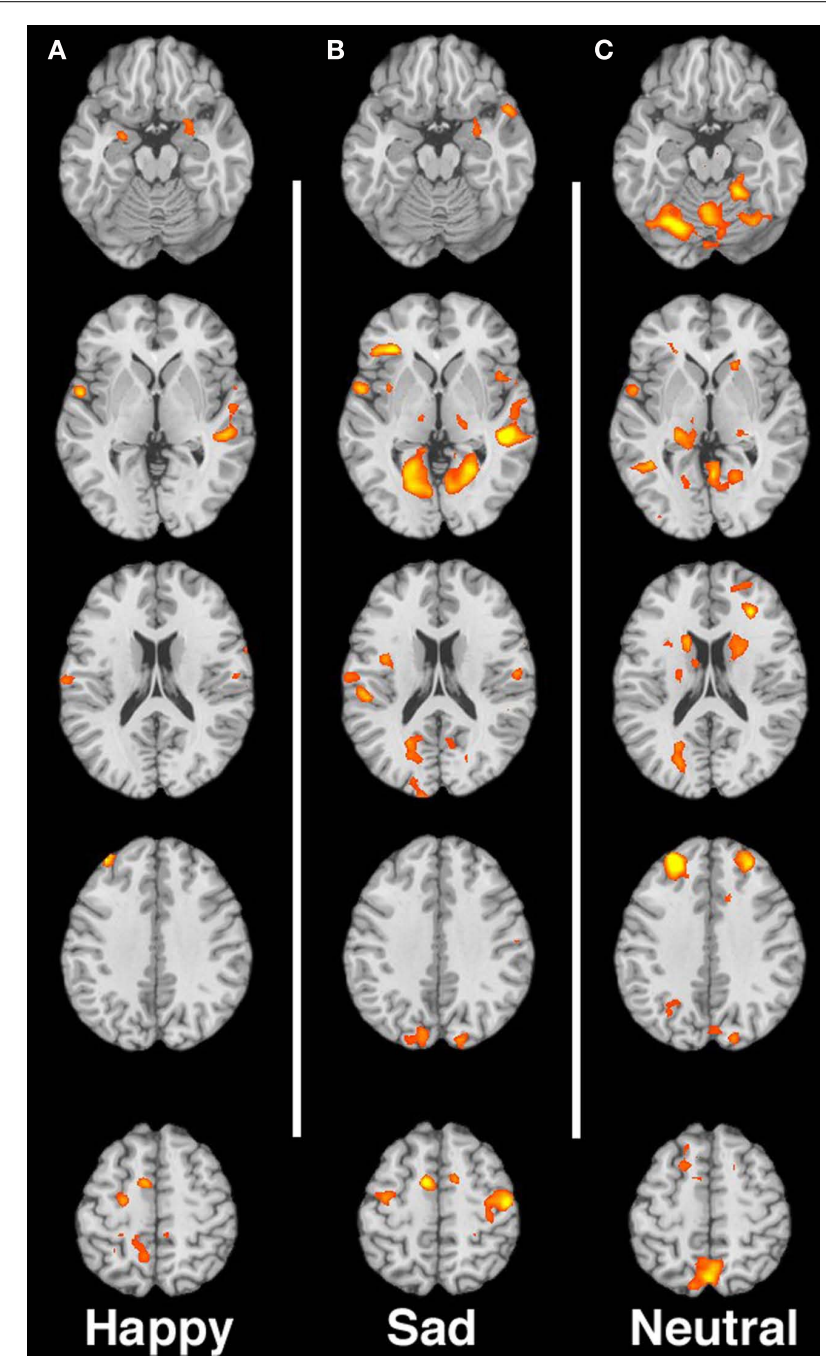

FIGURE 1 | (A-C) Contrast of non-using > substance-using mothers for happy, sad, and neutral faces, respectively. Images are shown in neurological convention, with the left hemisphere on the left side of each image. Slices are at MNI $z$-coordinate locations $-19,-1,+17,+35$, and $+53 \mathrm{~mm}$.

Substance-using mothers tended to show less activation in response to sad infant faces. In this condition, we observed greater activation for non-using relative to substance-using mothers in extensive regions of cortex, including prefrontal regions such as the right and left dlPFC/MFG, medial orbitofrontal cortex, right inferior frontal gyrus (IFG), sensorimotor regions, the middle/superior temporal gyrus and posterior cingulate cortex (PCC). In addition we observed greater activation for non-users in visual processing regions such as the right occipital gyrus/cuneus, and in limbic regions, including the right amygdala and parahippocampal gyrus (see Table 3). There were no areas that showed greater activation for substance-using relative to non-using mothers.

\section{Neutral expressions}

For the neutral faces, we again observed greater activity for the non-using relative to the substance-using in several prefrontal 


\begin{tabular}{|c|c|c|c|c|c|}
\hline \multirow[t]{2}{*}{$\boldsymbol{x}$} & $y$ & $z$ & $\boldsymbol{k}$ & Max & Region \\
\hline & & & & & $\mathrm{NU}>\mathrm{SU}$ \\
\hline 10 & -39 & 63 & 57 & 4.314 & R. postcentral gyrus/superior parietal \\
\hline 32 & 37 & 26 & 48 & 3.974 & R. middle frontal gyrus/dIPFC \\
\hline-8 & 14 & 54 & 50 & 3.923 & $\begin{array}{l}\text { L. medial/superior frontal } \\
\text { gyrus/dmPFC }\end{array}$ \\
\hline 7 & 42 & -12 & 46 & 3.811 & R. vmPFC \\
\hline 30 & -89 & 3 & 28 & 3.683 & R. middle occipital gyrus \\
\hline 27 & -34 & 2 & 57 & 3.591 & R. hippocampus/parahippocampus \\
\hline-29 & -71 & -12 & 35 & 3.220 & $\begin{array}{l}\text { L. cerebellum (declive) } \\
\text { SU > NU }\end{array}$ \\
\hline-34 & -55 & 6 & 26 & -4.358 & L. posterior parahippocampal gyrus \\
\hline
\end{tabular}

regions including the $\mathrm{dmPFC} /$ medial and superior frontal gyri, vmPFC, dIPFC, and right IFG, in sensorimotor regions, visual processing areas such as the cuneus/PCC and in limbic/striatal regions including the right amygdala/parahippocampal gyrus/globus pallidus (see Table 4). Again, there were no areas that showed greater activation for substance-using relative to non-using mothers.

\section{CRIES}

Figures 2A,B shows the neural response for the contrast of non-using mothers $>$ substance-using mothers for high and lowdistress cries respectively.

\section{Low-distress cries}

In response to low-distress infant cries, non-using mothers showed greater activation in auditory sensory processing regions including right superior/middle temporal gyri, and prefrontal regions such as the medial frontal gyrus/pre-SMA, sensorimotor regions, as well as regions involved in emotional processing, memory and empathy such as the insula, thalamus, and bilateral amygdala/parahippocampal gyrus (See Table 5).

\section{High-distress cries}

In response to high-distress cries, fewer regions differentiated non-using from substance-using groups. Increased activation for non-using relative to substance-using mothers was seen in the left superior/MFG, sensorimotor regions, insula, mid cingulate gyrus/precuneus and bilateral amygdala/parahippocampal gyrus (see Table 6; see also Table 7 for neural response to the tone).

\section{DISCUSSION}

In the study presented here, we used fMRI to investigate whether substance use during pregnancy or in the recent post-partum relates to neural response to infant cries and faces in post-partum mothers. We found generally reduced activation for substanceusing mothers relative to non-using mothers when processing such infant-related sensory stimuli in areas that have previously been identified in parenting studies and emotional processing more generally. To our knowledge, this study provides the first empirical evidence to suggest that the neural circuitry recruited when processing infant faces and cries is altered in mothers who use substances of abuse.

Across happy, sad, and neutral face conditions, we observed greater activity for non-using relative to substance-using mothers in prefrontal regions (e.g., dlPFC, vmPFC), visual processing regions (e.g., occipital cortex) and limbic regions (e.g., hippocampus/amygdala). These regions comprise a network underscoring social, emotional and visual sensory processing, and have been previously implicated in studies exploring the neural response to facial affect (e.g., Hariri et al., 2000; Gur et al., 2002). Additionally, many of these regions overlap with those observed to be active in previous studies of parental response to infant faces such as the medial prefrontal cortex, occipital cortex, hippocampus and amygdala (e.g., Strathearn et al., 2008). However, we also note that some regions previously identified in response to infant faces, particularly in the striatum (e.g., the caudate and putamen) did not discriminate substance-using from non-using mothers in our study. Nevertheless, the generally reduced activity in substanceusing relative to non-using mothers in response to infant facial expression of emotion supports the hypothesis that the neural systems associated with emotional processing of infant cues in substance-using mothers may be less responsive relative to those in non-using mothers.

With respect to cries, we also observed a large network of sensory and emotional processing regions that were more active for non-using mothers relative to substance-using mothers. For both low and high-distress cries we observed greater activation for non-using relative to substance-using mothers in auditory sensory processing regions (STG/MTG) as well as in sensorimotor/precentral gyrus, prefrontal and limbic regions (amygdala and parahippocampus bilaterally), as well as the insula. Again, many of these areas, including the amygdala, insula, MTG/STG as well as prefrontal regions, overlap with those previously identified as active for mothers in responses to infants cries (Swain et al., 2003, 2004a). Notably, we did not observe substance-related differences in all previously identified regions associated with cry perception (e.g., ACC). As with our findings for faces, these data indicate potential alterations in neural systems responsible for processing infant cries in substance-using mothers. These findings are consistent with the behavioral literature of negative parenting outcomes in situations of substance use, and may help to explain why appropriate interaction with the infant may be difficult for substance-using mothers.

Taken together, the data suggest generally reduced neural responsiveness to infant cues in substance-using compared to nonusing mothers. We suggest that such reduced neural responsiveness may lead to difficulty in subsequent behavioral maternal response to the infant, and in the formation of infant-caregiver attachment. In turn, this resulting difficulty may underlie some of the parenting difficulties observed in substance-using parents. Specifically, we suggest that reduced activation may reflect reduced saliency of infant cues themselves, which may lead to late or inappropriate parental response to the infants needs. This in turn could lead to a consequent failure to comfort the infant and thus an impaired ability to build an appropriate infant-caregiver relationship. In this way, substance use may lead to a cycle of inappropriate behavioral and neurobiological responses, involving altered neurobiological 
Table $3 \mid x, y, z$ coordinates, max activation, and cluster size for the contrast of non-using $>$ substance-using mothers for sad infant faces.

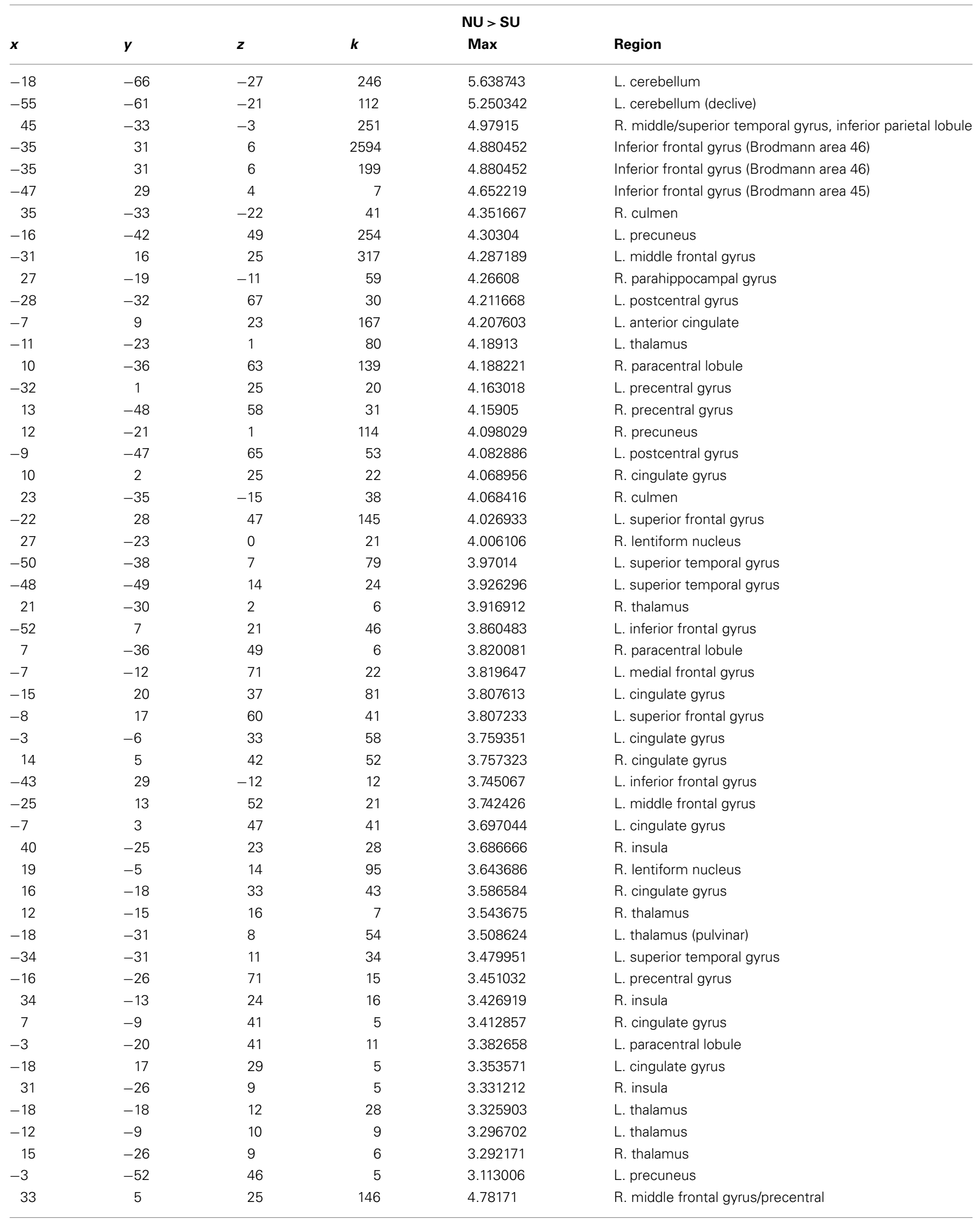




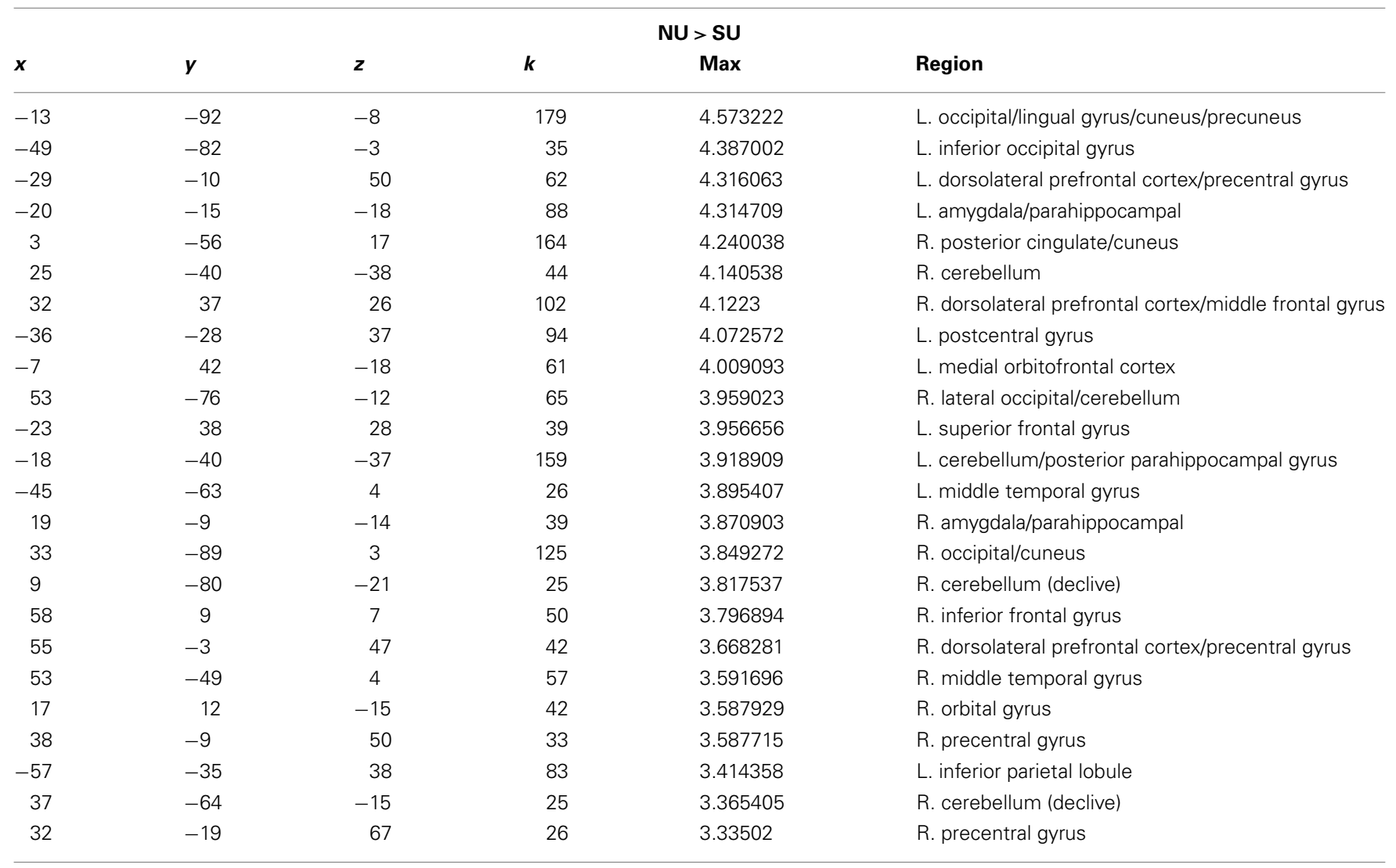

responses to infant sensory stimuli early in motherhood. Indeed extant work has demonstrated that the ability of mothers to detect signal differences in cries at the sensory level is important for providing an appropriate response to infant actions, which has implications for mother-child attachment (e.g., Donnovan et al., 2007).
Somewhat surprisingly, we found almost no regions that were more active for substance-using relative to non-using mothers. We might have expected that substance-using mothers would have shown heightened activation in stress related circuits, reflecting the idea that infant cues are less rewarding and more stressful for substance-using mothers, particularly for negative or

Table $4 \mid x, y, z$ coordinates, max activation, and cluster size for the contrast of non-using > substance-using mothers for neutral infant faces.

\begin{tabular}{ccccll}
\hline $\boldsymbol{x}$ & $\boldsymbol{y}$ & $\boldsymbol{z}$ & \multicolumn{2}{c}{ Max } & $\begin{array}{c}\text { NU } \mathbf{S U} \\
\text { Region }\end{array}$ \\
\hline-12 & 20 & 49 & 153 & 4.883 & L. medial/superior frontal gyrus \\
37 & -23 & 61 & 231 & 4.463 & R. precentral/postcentral gyus; SMA \\
9 & -75 & 24 & 475 & 4.448 & R. posterior cingulate cortex/cuneus \\
-40 & 27 & 4 & 168 & 4.318 & L. inferior frontal gyrus \\
-7 & 40 & -12 & 145 & 4.145 & L. ventromedial prefrontal cortex/medial orbitofrontal gyrus, subgenual ACC \\
3 & -36 & 52 & 328 & 4.004 & R. postcentral gyrus/mid cingulate cortex, precuneus \\
-16 & 44 & 30 & 35 & 3.810 & L. superior/medial frontal gyrus, dmPFC \\
7 & 5 & 25 & 31 & 3.746 & R. mid cingulate gyrus \\
-34 & -60 & -15 & 42 & 3.673 & L. cerebellum (declive) \\
36 & -80 & 28 & 28 & 3.669 & R. superior occipital gyrus \\
11 & 11 & 52 & 31 & 3.631 & R. medial frontal gyrus \\
-32 & -66 & -31 & 35 & 3.627 & L. cerebellum \\
-49 & -1 & 38 & 26 & 3.584 & L. dlPFC \\
36 & 17 & -20 & 47 & 3.529 & R. inferior frontal gyrus/superior temporal gyrus \\
-61 & -37 & 7 & 29 & 3.389 & L. posterior middle temporal gyrus \\
25 & -9 & -1 & 28 & 3.379 & R. amygdala/parahippocampal/globus pallidus
\end{tabular}




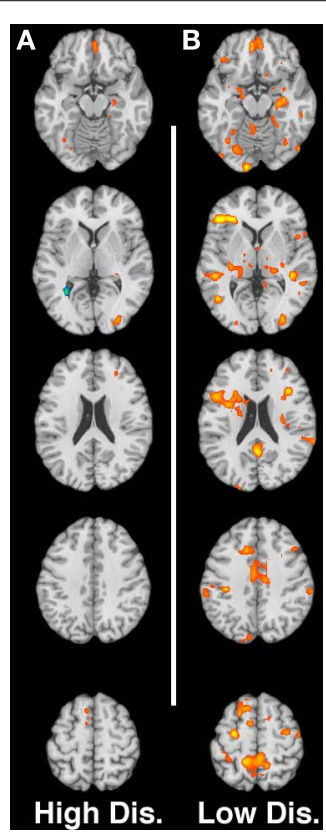

FIGURE 2 | (A,B) Contrast of non-using > substance-using for low-distress and high-distress cries respectively. Slices are at MNI $z$-coordinate locations $-16,+2,+20,+38$, and $+56 \mathrm{~mm}$.

upsetting stimuli (e.g., cries). For example, Sinha et al. (2005) have found that cocaine dependant but currently abstinent participants showed increased activation of corticolimbic circuitry (caudate and dorsal striatum) for stressful stimuli relative to healthy controls. Instead, we observed only one small region of increased activity in the left parahippocampus for happy faces. This null finding may be due to the nature of the stimuli and the populations being explored; that is, response to emotional or stressful stimuli may be particularly blunted in the presence of substance use, possibly via the mechanism of co-opting of motivation/reward circuitry. Alternatively, it is possible that the system is simply less responsive overall, or that the pattern of responding in substanceusing mothers is far more variable; future research will be necessary to directly address these possibilities. Such research could possibly include images that are likely to elicit increased response in substance users, such as paraphernalia, or stimuli that are more stressful than infant cries.

One of the important limitations of the present study is the heterogeneity of substances that were used by the mothers in our sample. Indeed the consequences of different substances of abuse vary at neurochemical as well as behavioral levels. In this sample, we are focusing on the impact of an addictive process on the response to what would be expected to be salient cues. Future studies should examine the potential impact of specific substances (and the quantity/frequency of their use) on the neural responses to infant stimuli, as well as investigate groups of parents (both mothers and fathers) with specific addictions. We also note differences between the groups in terms of maternal education and number of children in the home, as well as racial distribution across the groups as potential limitations of the current study; these factors are very difficult to match in studies of substance use, particularly in the case of SES/maternal education and race, as substance users tend to have lower SES than non-users, and are more often members of minority groups. We acknowledge that these factors may influence the results as they may influence home environments and parenting styles and thus should be examined further in future research. Nevertheless, the result of this study represent an important and novel step in identifying the impact of substance use on maternal responding.

Table 5 | $x, y, z$ coordinates, max activation, and cluster size for the contrast of non-using > substance-using mothers for low-distress infant cries.

\begin{tabular}{rccrll}
\hline \multicolumn{1}{c}{$\boldsymbol{x}$} & $\boldsymbol{y}$ & $\boldsymbol{k}$ & \multicolumn{1}{c}{$\mathbf{N U}>\mathbf{S U}$} \\
\hline 50 & -37 & 2 & 227 & 4.927 & Region \\
-24 & -4 & -17 & 48 & 4.734 & R. superior/middle temporal gyrus \\
-34 & 28 & 6 & 108 & 4.604 & L. inferior frontal gyrus \\
-11 & 3 & 50 & 75 & 4.328 & L. medial frontal gyrus/pre-SMA/dorsal cingulate gyrus \\
22 & -59 & 3 & 523 & 4.254 & R. lingual gyrus/fusiform gyrus/cuneus/middle occipital gyrus \\
-52 & -30 & 21 & 83 & 4.252 & Posterior insula/postcentral gyrus \\
51 & -17 & 49 & 247 & 4.228 & R. postcentral gyrus \\
-18 & -53 & -4 & 647 & 4.158 & L. lingual gyrus/fusiform gyrus/posterior parahippocampal/cuneus/middle occipital gyrus \\
-57 & -1 & 6 & 119 & 4.023 & L. mid/anterior insula/precentral gyrus \\
51 & -68 & -6 & 29 & 4.017 & R. lateral middle occipital gyrus \\
53 & 8 & -12 & 219 & 3.955 & R. amygdala/parahippocampal/middle temporal/superior temporal gyrus \\
-37 & -11 & 43 & 145 & 3.893 & L. precentral gyrus \\
-18 & -20 & 9 & 24 & 3.818 & L. thalamus \\
-19 & -29 & 67 & 32 & 3.779 & L. precentral gyrus \\
15 & -21 & 7 & 29 & 3.718 & R. thalamus \\
25 & -35 & 44 & 29 & 3.543 & R. postcentral gyrus \\
47 & 7 & 6 & 24 & 3.524 & R. insula \\
14 & 2 & 47 & 60 & 3.397 & R. medial frontal gyrus/pre-SMA/dorsal cingulate/gyrus
\end{tabular}


Table $6 \mid x, y, z$ coordinates, max activation, and cluster size for the contrast of non-using $>$ substance-using mothers for high-distress infant cries.

\begin{tabular}{|c|c|c|c|c|c|}
\hline \multicolumn{6}{|c|}{$\mathbf{N U}>\mathbf{S U}$} \\
\hline$x$ & $y$ & $z$ & $\boldsymbol{k}$ & Max & Region \\
\hline-41 & 42 & 33 & 27 & 4.459 & L. superior/middle frontal gyrus/dIPFC \\
\hline 50 & -34 & 0 & 65 & 4.219 & R. middle temporal gyrus \\
\hline-64 & -19 & 15 & 29 & 4.107 & L. postcentral gyrus \\
\hline 62 & -25 & 20 & 49 & 3.923 & R. postcentral gyrus \\
\hline 66 & -1 & 16 & 35 & 3.683 & R. precentral gyrus \\
\hline-11 & 3 & 50 & 39 & 3.630 & L. medial frontal gyrus/pre-SMA/dorsal cingulate gyrus \\
\hline 28 & 0 & -12 & 49 & 3.540 & R. amygdala/parahippocampal gyrus \\
\hline 53 & -20 & 3 & 33 & 3.538 & R. superior temporal gyrus \\
\hline
\end{tabular}

Table $7 \mid x, y, z$ coordinates, max activation, and cluster size for the contrast of non-using $>$ substance-using mothers for the pure tone.

\begin{tabular}{|c|c|c|c|c|c|}
\hline \multicolumn{6}{|c|}{$\mathbf{N U}>\mathbf{S U}$} \\
\hline$x$ & $y$ & $z$ & $\boldsymbol{k}$ & Max & Regions \\
\hline-29 & 43 & 31 & 168 & 5.294 & L. superior frontal gyrus \\
\hline-21 & -29 & -1 & 247 & 4.975 & L. thalamus/midbrain/posterior cingulate \\
\hline-7 & -80 & -6 & 1583 & 4.694 & L. cerebellum/lingual/occipital gyrus \\
\hline-8 & 11 & 66 & 64 & 4.426 & L. superior/medial frontal gyrus \\
\hline 25 & 7 & 60 & 97 & 4.247 & R. superior/anterior cingulate gyrus \\
\hline-43 & -52 & 1 & 40 & 4.072 & L. inferior temporal gyrus \\
\hline-20 & 14 & 14 & 87 & 3.989 & L. dorsal caudate \\
\hline 23 & 10 & 17 & 132 & 3.930 & R. dorsal caudate \\
\hline-22 & -12 & 17 & 25 & 3.316 & Thalamus \\
\hline-22 & 14 & 49 & 37 & 3.313 & Superior frontal gyrus \\
\hline
\end{tabular}

It will be important for future work to further explore individual differences among substance-using and non-using mothers in order to better understand the underlying contributing factors responsible for behavioral and neurobiological differences associated with parenting under conditions of substance use. For instance, substance-using mothers report higher levels of stress than non-substance-using mothers (Kelley, 1998), and maternal stress is considered to be an important mediator of parenting, as well as child outcomes (Suchman and Luthar, 2001). Substanceabusing mothers may also be more likely to have undergone emotional or physical trauma and like stress, this may impact their parenting. Future studies investigating possible contributions related to stress and trauma, as well as domains that might be influenced by stress and trauma exposure (e.g., mood and attention) warrant direct investigation. Moreover, additional research on the neural circuitry associated with response to emotion under conditions of substance use/addiction in mothers will be necessary to determine whether our observed findings are specific to infant emotion and social cues or reflect a more general reduced sensitivity in circuits involved in emotion processing. Because the current investigation focused on infant emotional stimuli, it is not possible to determine if the observed effects of substance use on brain activation would generalize to other types of relevant stimuli. One additional area for future exploration, which has been explored in non-abusing parents, is the degree to which processing of images or cries of one's own infant mediates neural responses. With respect to substance use, it would be helpful to determine whether some of the differences we observed between substance 
users and non-users are alleviated when mothers view their own relative to an unknown infant; it is possible that viewing or listening to one's own infant will be more salient, thus mitigating our observed altered response. Exploring these factors along with better understanding the specificity of the response to infant emotion/cues compared to other social/emotional cues and the effects of specific drugs and degrees of addiction will go a long way toward furthering our understanding of parenting under conditions of substance use and abuse.

Finally, one important implication from our findings of altered neural circuitry in response to infant cues in substance-using post-partum mothers is that interventions that focus on improving mother-child interactions rather than simply on eliminating substance use may be equally important for improving motherchild attachment in the presence of substance use. Specifically, increasing the amount and quality of appropriate mother-child interaction in the post-partum may help to retune the circuitry involved in reward/motivation and parenting. Recent work focusing on mother-child interactions in substance-using mothers to increase maternal mindfulness of the baby has had positive results, including improvements in observed mother-toddler interactions (e.g., Suchman et al., 2008). Future work that examines functional neural activity before and after this style of intervention would be useful for determining whether improvements in observed behavior correspond with changes in the underlying neural circuitry and more generally if there is significant plasticity in the neural circuitry for parenting in substance-abusing mothers.

\section{ACKNOWLEDGMENTS}

This work was supported by the NIH (NIDA) grants P01 DA022446 and R01 DA026437. This publication was also made possible by CTSA Grant Number UL1 RR024139 from the National Center for Research Resources (NCRR), a component of the National Institutes of Health (NIH), and NIH roadmap for Medical Research. Its contents are solely the responsibility of the authors and do not necessarily represent the official view of any of the funding agencies. The authors report that they have no financial conflicts of interest with respect to the content of this manuscript. Dr. Potenza has received financial support or compensation for the following: Dr. Potenza has consulted for and advised Boehringer Ingelheim; has consulted for and has financial interests in Somaxon; has received research support from the National Institutes of Health, Veteran's Administration, Mohegan Sun Casino, the National Center for Responsible Gaming and its affiliated Institute for Research on Gambling Disorders, and Forest Laboratories, Ortho-McNeil, Oy-Control/Biotie, Glaxo-SmithKline and Psyadon pharmaceuticals; has participated in surveys, mailings or telephone consultations related to drug addiction, impulse control disorders or other health topics; has consulted for law offices and the federal public defender's office in issues related to impulse control disorders; provides clinical care in the Connecticut Department of Mental Health and Addiction Services Problem Gambling Services Program; has performed grant reviews for the National Institutes of Health and other agencies; has guest-edited journal sections; has given academic lectures in grand rounds, CME events and other clinical or scientific venues; and has generated books or book chapters for publishers of mental health texts. The other authors reported no biomedical financial interests or other conflicts of interest. We also thank Dr. James Green for sharing his cry stimuli with us, Max Greger-Moser for assisting with collection of cry rating data, Marion Mayes for coordinating visits and working with participants and Kara Holcomb for overseeing the fMRI visits with participants.

\section{REFERENCES}

Asenio, S., Romero, M. J., Palu, C., Sanchez, A., Senebre, I., Morales, J., Carcelen, R., and Romero, F. (2010). Altered neural response of the appetitive emotional system in cocaine addiction:an fRMI study. Addict. Biol. 15, 504-516.

Ashburner, J., and Friston, K. J. (2005). Unified segmentation. Neuroimage 26, 839-851.

Bartels, A., and Zeki, S. (2004). The neural correlates of maternal and romantic love. Neuroimage 21, 1155-1166.

Breiter, H. C., Gollub, R. L., Weisskoff, R. M., Kennedy, D. N., Makris, N., Berke, J. D., Goodman, J. M., Kantor, H. L., Gastfriend, D. R., Riorden, J. P., Mathew, R. T., Rosen, B. R., and Hyman, S. E. (1997). Acute effects of cocaine on human brain activity and emotion. Neuron 19, 591-611.

Chambers, R. A., Bickel, W. K., and Potenza, M. N. (2007). A scale-free systems theory of motivation and addiction. Neurosci. Biobehav. Rev. 31, 1017-1045.

Childress, A. R., Mozley, P. D., McElgin, W., Fitzgerald, J., Reivich, M., and O'Brien, C. P. (1999). Limbic activation during cue-induced cocaine craving. Am. J. Psychiatry 156, 11-18.

Donnovan, W., Leavitt, L., Taylor, N., and Border, J. (2007). Maternal sensory sensitivity, motherinfant 9-month interacton, infant attachment status: predictors of mother-toddler interaction at 24 months. Infant Behav. Dev. 30, 336-352.

Everitt, B. J., and Robbins, T. W. (2005). Neural systems of reinforcement for drug addiction: from actions to habits to compulsion. Nat. Neurosci. 8, 1481-1489.

Gottwald, S. R., and Thurman, S. K. (1994). The effects of prenatal cocaine exposure on mother-infant interaction and infant arousal in the newborn period. Topics Early Child. Spec. Educ. 14, 217-231.
Green, J. A., and Gustafson, G. E. (1983). Individual recognition of human infants on the basis of cries alone. Dev. Psychobiol. 16, 485-493.

Gur, R. C., Schroeder, L., Turner, T., McGrath, C., Chan, R. M., Turetsky, B. I., Alsop, D., Maldjian, J., and Gur, R. E. (2002). Brain activation during facial emotion processing. Neuroimage 16, 651-662.

Hariri, A. R., Bookheimer, S. Y., and Mazziotta, J. C. (2000). Modulating emotional responses: effects of a neocortical network on the limbic system. NeuroReport 11,43-48.

Johnson, A. L., Morrow, C. E., Accornero, V. H., Xue, L., Anthony, J. C., and Bandstra, E. S. (2002). Maternal cocaine use: estimated effects on mother-child play interactions in the preschool period. J. Dev. Behav. Pediatr. 23, 191-202.

Kelley, S. J. (1998). Stress and coping behaviors of substance-abusing mothers. J. Soc. Pediatr. Nurs. 3, 103-110.
Kober, H., Mende-Siedlecki, P., Kross, E., Weber, J., Mischel, W., Hart, C., and Ochsner, K. N. (2010). Prefrontal striatal pathway underlies cognitive regulation of craving. Proc. Natl. Acad. Sci. U.S.A. 107, 14811-14816. Leibenluft, E., Gobbini, M. I., Harrison, T., and Haxby, J. V. (2004). Mothers' neural activation in response to pictures of their children and other children. Biol. Psychiatry 56, 225-232.

Lorberbaum, J. P., Newman, J. D., Horwitz, A. R., Dubno, J. R., Lydiard, R. B., Hamner, M. B., Bohning, D. E., and George, M. S. (2002). A potential role for thalamocingulate circuitry in human maternal behavior. Biol. Psychiatry 51, 431-445.

Maas, L. C., Lukas, S. E., Kaufman, M. J., Weiss, R. D., Daniels, S. L., Rogers, V. W., Kukes, T. J., and Renshaw, P. F. (1998). Functional magnetic resonance imaging of human brain activation during cue-induced cocaine craving. Am. J. Psychiatry 155,124-126. 
Mayes, L. C., Feldman, R., Granger, R. H., Haynes, O. M., Bornstein, M. H., and Schottenfeld, R. (1997). The effects of polydrug use with and without cocaine on mother-infant interaction at 3 and 6 months. Infant Behav. Dev. 20, 489-502.

Mayes, L. C., and Sean T. D. (2002). "Substance abuse and parenting," in Handbook of Parenting. Vol. 4. Social Conditions and Applied Parenting, 2nd Edn, ed. M. H. Bornstein (Mahwah, NJ: Lawrence Erlbaum Associates Publishers), 329-359.

Molitor, A., and Mayes, L. C. (2010). Problematic dyadic interaction among toddlers and their polydrugcocaine-using mothers. Infant Ment. Health J. 31, 121-140.

Murray, L. (1992). The impact of postnatal depression on infant development. J. Child Psychol. Psychiatry 33, 543-561.

Network NICHD Early Child Care Research. (1999). Child care and mother-child interaction in the first 3 years of life. Dev. Psychol. 35, 1399-1413.

Nitschke, J. B., Nelson, E. E., Rusch, B. D., Fox, A. S., Oakes, T. R., and Davidson, R. J. (2004). Orbitofrontal cortex tracks positive mood in mothers viewing pictures of their newborn infants. Neuroimage 21, 583-592.

Rutherford, H., Williams, S., Moy, S., Mayes, L., and Johns, J. (2011). Disruption of maternal parenting circuitry by addictive process: rewiring of reward and stress systems.

Seifritz, E., Esposito, F., Neuhoff, J. G., Luthi, A., Mustovic, H., Dammann, G., von Bardeleben, U., Radue, E. W., Cirillo, S., Tedeschi, G., and Di Salle, F. (2003).
Differential sex-independent amygdala response to infant crying and laughing in parents versus nonparents. Biol. Psychiatry 54, 1367-1375.

Sinha, R., Lacadie, C., Skudlarski, P., Fulbright, R. K., Rounsaville, B. J., Kosten, T. R., and Wexler, B. E. (2005). Neural activity associated with stress-induced cocaine craving: a functional magnetic imaging study. Psychopharmacology (Berl.) 183, 171-180.

Strathearn, L., Li, J., Fonagy, P., and Montague, P. R. (2008). What's in a smile? Maternal brain responses to infant facial cues. Pediatrics 122, 40-51.

Strathearn, L., and McClure, S. M. (2002). A Functional MRI Study of Maternal Responses to Infant Facial Cues. Program No. 517.2.2002 Abstract Viewer/Itinerary Planner, Washington, DC: Social Neuroscience. [Abstract Online].

Suchman, N., Decoste, C., Castiglioni, N., Legow, N., and Mayes, L. (2008). The mothers and toddlers program: preliminary findings from an attachment-based parenting intervention for substanceabusing mothers. Psychoanal. Psychol. 25, 499-517.

Suchman, N. E., and Luthar, S. S. (2001). The mediating role of parenting stress in methadone-maintained mothers' parenting. Parent. Sci. Pract. 1, 285-315.

Swain, J. E., Leckman, J. F., Mayes, L. C., Feldman, R., Constable, R. T., and Schultz, R. (2003). "The neural circuitry of parent-infant attachment in the early postpartum," in American College of Neuropsychopharmacology 42nd Annual Meeting, San Juan.
Swain, J. E., Leckman, J. F., Mayes, L. C., Feldman, R., Constable, R. T., and Schultz, R. T. (2004a). Neural substrates of human parent-infant attachment in the postpartum. Biol. Psychiatry 55, 153s.

Swain, J. E., Mayes, L. C., and Leckman, J. F. (2004b). The development of parent-infant attachment through dynamic and interactive signaling loops of care and cry. Behav. Brain Sci. 27, 472-473.

Swain, J. E., and Lorberbaum, J. P. (2008). "Imaging the parental brain," in Neurobiology of the Parental Brain, ed. R. S. Bridges (London: Elsevier), 83-110.

Swain, J. E., Lorberbaum, J. P., Kose, S., and Strathearn, L. (2007). Brain basis of early parent-infant interactions: psychology, physiology, and in vivo functional neuroimaging studies. J. Child Psychol. Psychiatry 48, 262-287.

Talairach, J., and Tornoux, P. (1988). Co-planar Stereotaxic Atlas of the Human Brain: 3-Dimensional Proportional System: An Approach to Cerebral Imaging. Stuttgart: Georg Thieme.

U.S. Dept. of Health and Human Services, National Center for Health Statistics, and National Health Interview Survey. (1999). ICPSR version. Hyattsville, MD: U.S. Dept. of Health and Human Services, National Center for Health Statistics 1999; Ann Arbor, MI: Interuniversity Consortium for Political and Social Research 2002.

Volkow, N. D., and Li, T. K. (2004). Drug addiction: the neurobiology of behaviour gone awry. Nat. Rev. Neurosci. 5, 963-970.

Wager, T. D., Keller, M. C., Lacey, S. C., and Jonides, J. (2005). Increased sensitivity in neuroimaging analyses using robust regression. Neuroimage 26, 99-113.

Wexler, B. E., Gottschalk, C. H., Fulbright, R. F., Prohovnik, I., Lacadie, C. M., Rounsaville, B. J., and Gore, J. C. (2001). fMRI of cocaine craving. Am. J. Psychiatry 158, 86-95.

Wolff, P. H. (1969). "The natural history of crying and other vocalizations in early infancy," in Determinants of Infant Behaviour IV, ed. B. M Foss (London: Methuen), 81-109.

Conflict of Interest Statement: The authors declare that the research was conducted in the absence of any commercial or financial relationships that could be construed as a potential conflict of interest.

Received: 03 March 2011; paper pending published: 12 April 2011; accepted: 22 May 2011; published online: 15 June 2011.

Citation: Landi N, Montoya J, Kober H, Rutherford HJV, Mencl WE, Worhunsky PD, Potenza MN and Mayes LC (2011) Maternal neural responses to infant cries and faces: relationships with substance use. Front. Psychiatry 2:32. doi: 10.3389/fpsyt.2011.00032

This article was submitted to Frontiers in Child and Neurodevelopmental Psychiatry, a specialty of Frontiers in Psychiatry. Copyright (C) 2011 Landi, Montoya, Kober, Rutherford, Mencl, Worhunsky, Potenza and Mayes. This is an openaccess article subject to a non-exclusive license between the authors and Frontiers Media SA, which permits use, distribution and reproduction in other forums, provided the original authors and source are credited and other Frontiers conditions are complied with. 


\section{APPENDIX \\ CRY ACOUSTIC PROPERTIES}

Although cries were not selected based on acoustic properties we report properties that have been observed to correlate with perceived distress including, pitch, number of bouts and duration of bouts, and number of inter-bout pauses and duration of inter-bout pauses. All cries had sampling frequencies of $44100 \mathrm{~Hz}$ and ranged from 1.9 to $2.12 \mathrm{~s}$. All cries were normalized to the same relative peak intensity using Praat software http://www.fon.hum.uva.nl/praat/. Presentation volume was occasionally adjusted for participant comfort. High-distress cry 1: minimum pitch $129.53 \mathrm{~Hz}$; maximum pitch 433.6; mean pitch
$351.27 \mathrm{~Hz}$; number of bouts 2; mean bout length 0.97 ; mean pause length 0.09 s. High-distress cry 2: minimum pitch $209.68 \mathrm{~Hz}$; maximum pitch 461.32; mean pitch $317.17 \mathrm{~Hz}$; number of bouts 1; mean bout length $2.1 \mathrm{~s}$; mean pause length $0 \mathrm{~s}$. Low-distress cry 1 : minimum pitch 297.548; maximum pitch $470.003 \mathrm{~Hz}$; mean pitch $348.77 \mathrm{~Hz}$; number of bouts 3 ; mean bout length $0.47 \mathrm{~s}$; number of pauses 3; mean pause length $0.75 \mathrm{~s}$. Low-distress cry 2: maximum pitch 469.824; mean pitch $351.40 \mathrm{~Hz}$; number of bouts 9; mean bout duration $0.11 \mathrm{~s}$; number of pauses 4 ; mean pause length $0.1 \mathrm{~s}$. For the $220-\mathrm{Hz}$ pure tone the sampling rate was $441000 \mathrm{~Hz}$; duration was $2 \mathrm{~s}$; average pitch was $220 \mathrm{~Hz}$. The pure tone had a $2 \mathrm{~ms}$ ramp up at the beginning and the end of the token. 